\title{
FUENTES DE VARIANZA E INDICES DE VARIANZA EXPLICADA EN LAS CIENCIAS DEL MOVIMIENTO HUMANO
}

\author{
José Moncada Jiménez, Andrea Solera Herrera, y Walter Salazar Rojas \\ Escuela de Educación Física y Deportes \\ Universidad de Costa Rica, San José, Costa Rica \\ E-mail: jmoncada@cariari.ucr.ac.cr
}

\begin{abstract}
Resumen
Moncada Jiménez, J., Solera Herrera, A., y Salazar Rojas, W. (2002). Fuentes de varianza e índices de varianza explicada en las ciencias del movimiento humano. Revista de Ciencias del Ejercicio y la Salud, 2(2), 70-74. El análisis de varianza (ANOVA) ha sido utilizado ampliamente para analizar datos de diseños experimentales en las ciencias del movimiento humano. En este trabajo se presentan y se discuten tres indices de varianza explicada: epsilon cuadrado, eta cuadrado y omega cuadrado. Epsilon cuadrado es una mejor estimación de la fuerza de la asociación de las variables en una población de lo que es eta cuadrado. Omega cuadrado y epsilon cuadrado son las dos opciones principales disponibles para los investigadores que utilizan ANOVA. Sin embargo, omega cuadrado posee la ventaja de que el índice ha sido extendido a diseños y modelos más complejos, y es mucho mejor conocido por los usuarios de ANOVA, de lo que es epsilon cuadrado. Por esa razón omega cuadrado es el índice preferido por los investigadores que trabajan con datos provenientes de experimentos. PALABRAS CLAVES: estadística, análisis de varianza, ciencias del movimiento.
\end{abstract}

\section{LA VARIANZA}

La palabra varianza es un constructo que cuantifica la naturaleza variable de la medición. De acuerdo con Salazar (2002), existen tres fuentes principales de varianza: la verdadera, la extraña y la de error.

La varianza verdadera. En algunos casos también llamada variancia primaria, sistemática o experiemental, es la causada por el efecto del tratamiento, es decir de la variable independiente (Hyllegard, Mood, y Morrow, 1996). Según Kerlinger (1998), en una investigación se desea MAXIMIZAR la varianza, por lo que el investigador procura: aplicar tratamientos intensivos, y de diferencias entre ellos grandes. Por ejemplo, si se desea estudiar el efecto de dos dosis de un suplemento nutricional sobre el rendimiento aeróbico, lo que se pretendería bajo la premisa anterior, es tener dosis muy diferentes del mismo suplemento (e.g., $10 \mathrm{mg}$ vs. $30 \mathrm{mg}$ ), en lugar de dosis muy similares (e.g., $15 \mathrm{mg}$ vs. $17 \mathrm{mg}$ ).

La varianza extraña o secundaria. Es la variación que ocurre en los valores de la medición producto de variables potencialmente independientes que van a afectar a la variable dependiente, pero que no han sido incluidas en el estudio. De acuerdo con Hyllegard et al. (1996), una báscula que añada 200 g a cada medición es una fuente de varianza extraña. Este instrumento de medición, calibrado inadecuadamente, consistentemente añadiría $200 \mathrm{~g}$ a las mediciones que se realicen, por lo que las lecturas serían siempre $200 \mathrm{~g}$ mayores de lo que realmente son. Si las fuentes de varianza extraña están presentes en todos los métodos de medición, entonces los resultados posiblemente sobreestimarán o subestimarán consistentemente los valores verdaderos de la variable dependiente.

Otro ejemplo podría ser que en un estudio donde se mida si determinado entrenamiento mejora el rendimiento físico, el investigador deje al azar si los participantes desayunan o no, por lo que las mediciones se pueden ver afectadas por una variable extraña, que no ha sido contemplada dentro del estudio.

Para tratar de REDUCIR la varianza extraña hay varias estrategias que se pueden seguir, como por ejemplo:

a. Utilizar la asignación aleatoria de sujetos a los grupos experimentales en los diseños 
de grupos independientes (i.e., diseños conocidos como entre-sujetos o "betweengroups"), así se le permite al investigador partir del supuesto de que los grupos son homogéneos en todas las variables desde el inicio; y también que los grupos están expuestos de manera similar a factores externos durante la realización del estudio (Hernández, Fernández y Baptista, 1995). Por ejemplo, si se desea trabajar con un grupo control que no recibe tratamiento, y uno experimental que sí lo recibe, lo más recomendable sería asignar los participantes aleatoriamente a estos grupos, ya que por esta técnica cualquier variable que se desee (edad, estatura, inteligencia, fatiga, calidad de la alimentación, etc), quedaría con valores similares en ambos grupos desde el inicio y durante el estudio; asegurándose así que las variaciones en las mediciones de la variable dependiente se van a deber al efecto del tratamiento.

b. Asignar aleatoriamente el orden de los tratamientos en los diseños de mediciones repetidas en cada sujeto (i.e., diseños conocidos como intra-sujetos o "withinsubjects"). Por ejemplo, en una investigación donde se desee saber si la velocidad de un corredor se ve afectada por su estado de deshidratación o euhidratación, el asignar los sujetos aleatoriamente a los tratamientos permite asegurar que un día caluroso -variable extraña al estudio- afecte por igual a los participantes que se encuentran deshidratados o rehidratados. Así también, en algunos casos, este procedimiento permite controlar el posible aprendizaje de las pruebas que se aplican, ya que de darse este sesgo, estaría afectando por igual, los resultados de ambos grupos (Campbell y Stanley, 1963).

c. Convertir la variable extraña potencial en una "constante", como sería el caso de estandarizar un mismo desayuno antes de comenzar con las mediciones de los participantes.

d. Incluir la variable extraña como una variable independiente más dentro del estudio, y de esta manera estudiar el efecto directo que esta puede producir sobre la variable dependiente (Salazar, 2002).

La varianza de error. La tercer fuente de varianza se refiere a la variación inconsistente en los valores de la medición de la variable dependiente. De acuerdo con Hyllegard et al. (1996), la varianza de error es una variación que ocurre de manera natural e inexplicable y que siempre está presente en las mediciones. Aún cuando el investigador piense que todas las variables posibles han sido controladas de la mejor manera, siempre existirá varianza de error. En términos prácticos, la varianza de error es considerada como la variación entre los puntajes de los sujetos.

La varianza de error según Kerlinger (1998) se debe MINIMIZAR, debido a que "cualquier aumento en la varianza de error disminuye el valor de la razón $F$ (de ANOVA) $\mathrm{y}$ reduce las posibilidades de rechazar la hipótesis nula; mientras que cualquier reducción de la varianza de error aumenta las posibilidades de rechazarla (la hipótesis nula)" (Keppel, 1982, p. 67). Para Salazar (2002), esta varianza puede disminuirse utilizando muestras que sean más homogéneas entre sí, y utilizando instrumentos de medición que tengan una alta confiabilidad.

\section{EL ANÁLISIS DE VARIANZA}

El análisis de varianza (ANOVA o ANDEVA), es un procedimiento que se utiliza para descomponer la varianza en sus diversas fuentes. El cálculo del ANOVA produce una razón $F$ ( $F$ por Sir Ronald A. Fisher, su inventor), la cual, junto con los grados de libertad y un valor alfa predeterminado o también llamado nivel de significancia, contribuyen a tomar la decisión de si se mantiene o se rechaza la hipótesis nula $\left(\mathrm{H}_{0}\right)$ de una investigación. Por ejemplo, cuando se rechaza la $\mathrm{H}_{0}$ en un ANOVA, se indica que existen diferencias estadísticamente significativas entre las medias aritméticas (promedios) de las muestras; es decir, una diferencia mucho mayor de la que se hubiera encontrado por casualidad. Sin embargo, con muestras muy grandes, estas diferencias 
estadísticamente significativas puede que tengan poco significado práctico (Hinkle, Wiersna, y Jurs, 1994). Es por ello que se han diseñado diversos índices que procuran explicar el grado de asociación relativa entre la variable dependiente y la variable independiente, el cuál es expresado en términos de porcentaje, los cuales se presentan en la siguiente sección.

\section{INDICES DE VARIANZA EXPLICADA}

En esta sección se presentan tres índices de varianza explicada: eta cuadrado, epsilon cuadrado y omega cuadrado. Básicamente los tres índices explican el mismo concepto, la asociación entre la variable independiente (o sus niveles) y la variable dependiente. A pesar de que su cálculo es distinto, estos índices varían en un rango que va de .00 a 1.00; el cual se puede multiplicar por 100 para obtener un indicador porcentual de la varianza explicada (Keppel, 1982).

Para explicar mejor cada uno de ellos, se partirá del siguiente estudio ficticio donde en un grupo de secundaria, se quiere determinar si las muchachas que pertenecen al club de fútbol, tienen un mayor salto vertical que las compañeras que no pertenecen al club. Para ello se utiliza la prueba de "Salto Sargent", obteniéndose el cuadro de ANOVA (Tabla 1).

Tabla 1.

Cuadro resumen de ANOVA

\begin{tabular}{|c|c|c|c|c|c|c|}
\hline Varianza & Fuente & G.l. & Fórmula & Suma de Cuadrados & Cuadrado Medio & F \\
\hline $\mathrm{Vv}$ & $\mathrm{A}$ & $2-1=1$ & 5405.6 & 115.6 & 115.6 & $5.07 *$ \\
\hline $\mathrm{Ve}$ & $\mathrm{S} / \mathrm{A}$ & $2(5-1)=8$ & 5588 & 182.4 & 22.8 & \\
\hline $\mathrm{Vt}$ & $\mathrm{T}$ & $2 \times 5-1=9$ & 5290 & 298 & & \\
\hline
\end{tabular}

$* \mathrm{p}<0.1$

Abreviaturas: Vv: varianza verdadera; Ve: varianza de error; Vt: varianza total; G.1.: grados de libertad

\section{Eta cuadrado $\left(\eta^{2}\right)$}

Yaremko, Harari, Harrison, y Lynn (1982), definen a eta cuadrado $\left(\eta^{2}\right)$ como "una medida de la magnitud del efecto del tratamiento" (p. 71). Por su parte, Vincent (1999), la define como "una estimación general del tamaño del efecto" (p. 165).

Keppel (1982), indica que éste índice, que también se conoce como $\mathrm{R}^{2}$, ha sido propuesto como índice de la fuerza relativa para datos provenientes de experimentos. Para los modelos de ANOVA, se calcula como:

$$
R^{2}=\frac{\text { SC efecto }}{\text { SC Total }}
$$

en donde SC = Suma de Cuadrados.

Para la corporación SPSS (1997), cuando se estudian modelos de ANOVA de una vía por medio de su paquete estadístico, $\eta^{2}$ es la proporción de varianza en la variable dependiente que es explicada por las diferencias entre los grupos; y se calcula por medio de la división de la SC entre grupos (between groups) y la SC total. Sin embargo, para diseños factoriales, el tamaño del efecto brinda un valor $\eta^{2}$ para cada efecto principal (main effect). Es así como $\eta^{2}$ describe la proporción de variabilidad total atribuible a un factor (o variable independiente).

En el caso del ejemplo antes mencionado el valor de eta cuadrado, sería:

$$
R^{2}=\frac{115.6}{298}=0.39
$$

Es decir, un $39 \%$ de la varianza es explicada por el efecto del tratamiento.

\section{Epsilon cuadrado $\left(\varepsilon^{2}\right)$}

El segundo índice de varianza explicada es el epsilon cuadrado $\left(\varepsilon^{2}\right)$, que según Keppel (1982), es una mejor estimación 
de la fuerza de la asociación en una población de lo que es $\eta^{2}$. Maxwell, Camp y Arvey (1981), han reportado algunas dificultades cuando usan $\varepsilon^{2}$ en experimentos factoriales, y no queda claro si se puede generalizar el uso de $\varepsilon^{2}$ en los diferentes diseños experimentales como sí se puede hacer con otros índices (e.g., omega cuadrado). forma:

El índice $\varepsilon^{2}$ se calcula de la siguiente

$$
\varepsilon^{2}=\frac{\text { SC efecto }-(\text { g.l. efecto })(C M \text { error })}{S C \text { Total }}
$$

en donde: $S C=$ la suma de cuadrados; g.l. = los grados de libertad; $C M=$ el cuadrado medio.

Utilizando el ejemplo de la Tabla 1, el valor de epsilon cuadrado sería:

$$
\varepsilon^{2}=\frac{115.6-(1)(22.8)}{298}=0.31
$$

Lo cual quiere decir, que según este índice de varianza explicada, los datos o puntajes de la prueba de salto alto variaron en un $31 \%$ debido al efecto del tratamiento, es decir, al hecho de pertenecer o no al club de fútbol.

\section{Omega al cuadrado $\left(\omega^{2}\right)$}

Un tercer indicador se conoce como omega al cuadrado $\left(\omega^{2}\right)$. Hinkle, Wiersna, y Jurs (1994) proporcionan una definición acerca de este indicador de la varianza explicada:

"Una medida de la fuerza de la asociación entre la variable dependiente $e$ independiente en un ANOVA es $\omega^{2}$, omega cuadrado. Omega cuadrado indica la proporción de varianza en la variable dependiente que es explicada por los niveles de la variable independiente" (p. 338).

Keppel (1982), indica que el índice omega cuadrado provee una medida de la fuerza relativa de una variable independiente. Cohen (1977), sugiere que un efecto grande de un experimento en las ciencias del comportamiento y de las ciencias sociales, sería $\omega^{2} \geq 15 \%$. Un efecto mediano o moderado sería $\omega^{2}=6 \%$, y un efecto pequeño $\omega^{2}=1 \%$.

Omega al cuadrado no es afectado por muestras de tamaño pequeñas, mientras que la significancia de una $F$ sí es afectada por el tamaño de una muestra pequeña. Por lo tanto $\omega^{2}$ es insensible a las variaciones en el tamaño de la muestra.

La fórmula para omega cuadrado es:

$$
\omega^{2}=\frac{\text { SC efecto }-(\text { g.l. efecto })(C M \text { error })}{S C \text { Total }+ \text { CM error }}
$$

en donde: $S C=$ la suma de cuadrados; g.l. = los grados de libertad; $C M=$ el cuadrado medio.

De esta forma, en el ejemplo de las niñas futbolistas, omega al cuadrado se obtendría así:

$$
\omega^{2}=\frac{115.6-(1)(22.8)}{298+22.8}=0.23
$$

Es decir, que los puntajes de la prueba de "Salto Sargent" variaron en un 23\% debido al efecto de la variable independiente, es decir del club de fútbol.

Con respecto a $\omega^{2}$, también se debe tomar en cuenta las siguientes consideraciones:

1. Si el resultado del cálculo de una ANOVA, la $F<1$, no se debe calcular $\omega^{2}$, ya que resultaría en un número negativo, el cual no se podría interpretar correctamente (Keppel, 1982).

2. De acuerdo con Keppel (1982), este índice puede ser usado en diseños experimentales más complejos en los que se utilizan ANOVAS de dos o más vías. Así, se pueden obtener los porcentajes de cada fuente de varianza explicada que componen el modelo, sin que al sumarse sobrepasen el 100\%; aspecto que no siempre se cumple al utilizar $\eta^{2}$ y $\varepsilon^{2}$.

\section{CONCLUSIONES}

En un experimento, se pretende que los valores de la variable dependiente varíen o 
fluctúen como resultado presupuesto de la manipulación y control de las variables independientes. A esta variación o fluctuación, conocida técnicamente como "varianza", se le puede desglosar en tres fuentes: la varianza verdadera, la varianza extraña, y la varianza de error. Según Kerlinger (1998), el investigador que trata de probar la relación entre dos o más variables, debe velar por cumplir el principio MAXMINCON, el cual quiere decir: maximizar la varianza verdadera, minimizar la varianza extraña, y controlar la varianza de error.

Una vez recolectados los datos, y aplicado los principios antes mencionados, al analizar los datos el investigador escoge con cuál índice de varianza determinará la fuerza de la relación entre las variables, a saber, $\eta^{2}$, el cual muchas veces es indicado como la opción por omisión por los mismos paquetes estadísticos (e.g., $\operatorname{SPSS}^{\circledR}$, versión 8.0.); $\varepsilon^{2}$, donde su uso parece destinarse al trabajo con poblaciones, $\mathrm{u} \omega^{2}$, el cual es más utilizado al realizarse estudios factoriales o experimentales (Keppel, 1982).

\section{REFERENCIAS}

Campbell, D. y Stanley, J. (1963). Experimental and Quasi-experimental Designs for Research. Chicago: Rand McNally.

Cohen, J. (1977). Statistical power analysis for the behavioral sciences. New York: Academy Press.

Hernández, R, Fernández, C, y Baptista, P. (1995) Metodología de la Investigación. México: McGrawHill.

Hinkle, D. E., Wiersna, W., y Jurs, S. G. (1994). Applied statistics for the behavioral sciences ( $3^{\text {rd }} \mathrm{Ed}$.). Boston, MA: Houghton Mifflin.

Hyllegard, R., Mood, D. P., y Morrow, J. R. Jr. (1996). Interpreting research in sports and exercise science. St. Louis, MO: Mosby-Year Book, Inc.

Keppel, G. (1982). Design and analysis: A researcher's handbook ( $2^{\text {nd }}$ ed.). Englewood Cliffs, NJ: PrenticeHall.

Kerlinger, F. D. (1998). Investigación del Comportamiento (3ra Ed.). México: McGraw Hill.

Maxwell, S. E., Camp, C. J., y Arvey, R. D. (1981). Measures of strength of association: A comparative examination. Journal of Applied Psychology, 66(5), 525-534.

Salazar Rojas, W. (2002). Principios del paradigma cuantitativo en la investigación educativa. Revista de Ciencias del Ejercicio y la Salud, 2(1), 61-71.

SPSS, Inc. (1997). SPSS Advanced statistics 7.5. Chicago, IL.: SPSS Inc.
Vincent, W. J. (1999). Statistics in kinesiology ( $2^{\text {nd }}$ ed.). Champaign, IL: Human Kinetics.

Yaremko, R. M., Harari, H., Harrison, R. C., \& Lynn, E. (1982). Reference handbook of research and statistical methods in psychology: for students and professionals. New York, NY: Harper \& Row. 